\title{
Advancements in satellite remote sensing for mapping and monitoring of alien invasive plant species (AIPs)
}

\author{
Lwando Royimani $^{\mathrm{a}, *}$, Onisimo Mutanga ${ }^{\mathrm{a}}$, John Odindi $^{\mathrm{a}}$, Timothy Dube ${ }^{\mathrm{b}}$, \\ Trylee Nyasha Matongera ${ }^{\mathrm{a}}$ \\ ${ }^{a}$ Discipline of Geography, School of Agricultural, Earth and Environmental Sciences, University of KwaZulu-Natal, P/Bag X01, Scottsville, Pietermaritzburg, 3209, South \\ Africa \\ ${ }^{\mathrm{b}}$ Geo-Information and Earth Observation, Institute for Water Studies, Department of Earth Science, University of the Western Cape, Private Bag X17, Bellville, 7535, South \\ Africa
}

\section{A R T I C L E I N F O}

\section{Keywords:}

Satellite remote sensing

Spatial

Spectral

Temporal

AIPs

Parametric

Non-parametric

\begin{abstract}
A B S T R A C T
Detecting and mapping the occurrence, spatial distribution and abundance of Alien Invasive Plants (AIPs) have recently gained substantial attention, globally. This work, therefore, provides an overview of advancements in satellite remote sensing for mapping and monitoring of AIPs and associated challenges and opportunities. Satellite remote sensing techniques have been successful in detecting and mapping the spatial and temporal distribution of AIPs in rangeland ecosystems. Also, the launch of high spatial resolution and hyperspectral remote sensing sensors marked a major breakthrough to precise characterization of earth surface feature as well as optimal resource monitoring. Although essential, the improvements in spatial and spectral properties of remote sensing sensors presented a number of challenges including the excessive acquisition and limited temporal resolution. Therefore, the use of high spatial and hyperspectral datasets is not a plausible alternative to continued and operational scale earth observation, especially in financially constrained countries. On the other hand, literature shows that image classification algorithms have been instrumental in compensating the poor spatial and spectral resolution of remote sensing sensors. Furthermore, the emergence of robust and advanced nonparametric image classification algorithms have been a major development in image classification algorithms. Therefore, to address the inevitable challenges arising with satellite sensor development technology it is necessary to explore the use of robust and advanced non-parametric image classifiers with data provided by the new generation of multispectral sensors with improved spatial and spectral resolutions. This will promote longterm and large scale mapping of AIPs, especially in financially constrained countries.
\end{abstract}

\section{Introduction}

Globally, Alien Invasive Plants (AIPs) pose significant threats to, among others, natural ecosystems (Gurevitch and Padilla, 2004), biodiversity (Gaertner et al. 2009; Higgins et al. 1999), forests (Peerbhay et al. 2016a), rangelands and agricultural productivity (Pimentel et al. 2005). Furthermore, AIPs are known to reduce native plant species richness (Gaertner et al. 2009), alter fire regimes and soil properties (Pejchar and Mooney, 2009) and homogenize biodiversity (Peerbhay et al. 2016c; Kimothi and Dasari, 2010; Joshi et al. 2004) of invaded landscapes. Experimental studies have reported excessive economic losses as a result of alien invasion (Karki, 2009; Ayele, 2007). For instance, in the United States alone, the environmental impacts of the alien invasion were estimated to be approximately 120 billion US dollars per annum (Pimentel et al. 2005). In Australia, parthenium invasion in prime grazing land costs the government about 16.8 million US dollars annually, while in India, forty percent crop production losses are attributed to AIPs (McConnachie et al. 2011). Lowe et al. (2000) presented a list of invasive species around the world. The list comprises of Caulerpa Seaweed (Caulerpa taxifolia), Siam weed (Chromolaena odorata), Strawberry guava (Psidium cattleianum), Tamarisk (Tamarix ramosissima) and the Yellow himalayan raspberry (Rubus ellipticus). Several other AIPs with global distribution like parthenium (P. hysterophorus) (Nigatu et al. 2010), Bugweed (Solanum mauritianum) (Peerbhay et al. 2015), Tamarix spp (Swayne et al. nd), Bracken fern (Pteridium) (Matongera et al. 2017; Singh et al. 2013) and Pinus spp (Forsyth et al. nd) have also been reported.

To mitigate AIPs spread, timely and accurate information on spatial

\footnotetext{
${ }^{*}$ Corresponding author.

E-mail address: Odindi@ukzn.ac.za (L. Royimani).
} 
and temporal distribution, as well as abundance is required (Peerbhay et al. 2016a). This information is necessary to enhance the understanding of trends and patterns in AIPs spread for improved decisionmaking, optimal resource management and stewardship. Traditionally, field surveys and aerial photographs have been used to collect data on AIPs (Zuberi et al. 2014; Ayele, 2007; Foxcroft et al. 2008; Crossman and Kochergen, 2002; Everitt et al. 1996). However, many studies (e.g. Peerbhay et al. 2016c; Evangelista et al. 2009) noted that such approaches are not sustainable due to excessive capital, time and labor required, especially for large-scale applications. Furthermore, the use of traditional approaches like surveys are hampered by accessibility to the regions of interest, particularly in remote areas (Matongera et al. 2016a, 2016b; Curatola Fernández et al. 2013). Satellite remote sensing, on the other hand, has increasingly gained popularity as a plausible alternative in AIPs mapping (Dorigo et al. 2012; Aitkenhead and Aalders, 2011; Gómez-Casero et al. 2010). Unlike traditional approaches, satellite remotely sensed data and techniques can be applied on large and remote geographical locations (Huang and Asner, 2009). Similarly, the repeated coverage possible with satellite remote sensing approaches allows for detection of plant phenology which is necessary for the detection of AIPs spread (Flood, 2013).

Numerous studies (e.g. Matongera et al. 2016a; Peerbhay et al. 2016c; Niphadkar and Nagendra, 2016; Rocchini et al. 2015; Bradley, 2014; Boyd and Foody, 2011; Huang and Asner, 2009; Lass et al. 2005; Joshi et al. 2004) have explored remote sensing approaches to optimize the detection and mapping of AIPs. For instance, Huang and Asner (2009) provided an overview of spatial, spectral and temporal sensor resolutions for the detection of AIPs based on structural and functional traits at various canopy levels. Similarly, Peerbhay et al. (2016c) explored the value of multisource remotely sensed data for optimal detection of structural and functional properties of AIPs in commercial forests, while Bradley (2014) investigated the value of spectral, structural and phenological attributes in detecting AIPs. Lass et al. (2005), on the other hand, explored the use of hyperspectral dataset in detecting AIPs. To the best of our knowledge, there is no study that has tried to understand the use of improved resolution data provided by the new generation multispectral sensors in concert with advanced and robust image classification algorithms for detection of AIPs. In our opinion, it is necessary that future studies examine this relationship to improve large-scale and long-term mapping of AIPs in developing countries, currently constrained by existing acquisition costs of high spatial and hyperspectral remote sensing data.

The present study explores the importance of using the advanced and robust non-parametric image classification algorithms on freely available improved spatial and spectral resolution data provided by the new generation of multispectral sensors to promote AIPs mapping at an operational scale. Firstly, this paper provides a background on the ecology, as well as the spatial distribution of AIPs around the world. Secondly, the paper discusses sensor vegetation spectral properties necessary for discriminating AIPs as well as other remote sensing techniques used in the identification of AIPs from native vegetation. Thirdly, the review explores the applications of different satellite remote sensing techniques, such as multispectral, hyperspectral and multisource dataset in detection and mapping of AIPs as well as their financial implication in relation to scale of application and mapping accuracy. Fourthly, the paper outlines the importance of image classification with a detailed comparison of parametric and non-parametric or advanced robust machine learning algorithms in enhancing the detection of AIPs using remotely sensed data. Furthermore, the paper also draws a synergy between the type of remotely sensed data and a chosen image classifier. Lastly, the paper highlights the challenges of using remote sensing in the detection and mapping of AIPs and suggests directions for future research.

\section{The ecology and spatial distribution of AIPs}

Broadly, the term AIPs is used to refer to plant species or sub-plant species growing outside their naturally occurring habitats, with a strong ability to survive, reproduce, disperse and subsequently displace native flora (Kimothi and Dasari, 2010; Shezi and Poona, 2010; Mack et al. 2000). Generally, AIPs have identical functional features, such as competitive aggression and increased encroachment on disturbed environments. In these areas, AIPs take advantage of reduced interspecies competition as soils are either left bare or the native flora are still at an early stage of rejuvenation (Le Maitre et al. 2002). Studies have noted for instance that parthenium establishes and naturalize on empty niches along roadsides, railway tracks, fallow agricultural lands and around buildings (McConnachie et al. 2011). Similarly, Peerbhay et al. (2015) reported that the exotic Bugweed invades pasture lands, river-banks, forest margins and plantations. Other studies e.g. Curatola Fernández et al. (2013) have also reported that areas disturbed by fire are often preferred by the Bracken fern while Dark (2004) found that areas close to roads have a high density of noxious species in California.

Furthermore, studies have shown that AIPs have the engineering ability to modify their newly invaded habitats (Peerbhay et al. 2016b; Bax et al. 2003), thereby making it more suitable for their exponential growth and distribution. For instance, the allelopathic chemicals produced by parthenium not only displace indigenous plants but also transform river banks, grasslands, floodplains and woodlands into monocultural shrublands (McConnachie et al. 2011). Nigatu et al. (2010), for instance, found that the allelopathic chemicals produced by AIPs can inhibit germination and growth of indigenous plant species, which can change the structure and type of vegetation, fauna and local climate. Also, the literature shows that invaded landscapes are more likely to remain dominated by one individual species for a very long time (Huang and Asner, 2009). Although literature highlights some positive ecological and economic impact of AIPs, such as the provision of habitat to local fauna (Matongera et al. 2016a) and provision of fuelwood and carbon assimilation (Shackleton et al. 2007), the recorded ecological destruction as a result of the alien invasion is farreaching. For instance, AIPs out-compete the indigenous plants for available natural resources (i.e. water, sunlight, nutrients, space), which are integral to growth and distribution (Dark, 2004). Furthermore, the literature shows that AIPs are unpalatable to livestock and game grazing (Everitt et al. 1995; Pyšek, 1998).

According to Kalusová et al. (2013) and Pyšek (1998), plant species that are highly invasive have their origin in Europe. Mack et al. (2000), attribute this to the early (1500s) European voyages that contributed significantly to the human-driven dispersion. Blossey and Notzold (1995), for instance, reported that the Purple loose strife (Lythrum salicaria L.), native to Eurasia, was introduced in North America during the 1800s. Turbelin et al. (2017) presents a global distribution of invasive species in two various ways, the actual number (Fig. 1a) and the normalized value of invasive species per country (Fig. 1b). Findings from their work shown that the economically developed countries (i.e. United States, Alaska, New Zealand and Australia) along with newly industrialized countries (i.e. South Africa, Brazil, India and China) are significantly invaded by exotic species. These findings concur with Mack's et al. (2000) argument that human-beings are the major agents of AIPs dispersion. Generally, the developed and developing areas are characterized by large volumes of in-migration (Todaro, 1969), especially for tourism and economic destinations hence facilitating the introduction of AIPs (see Fig. 2).

On the other study, Richardson and Rejmanek (2011) reported that Australia, southern Africa, North America, Pacific Islands, Europe and New Zealand are the regions highly colonised by one or more of these invasive woody species, Acacia farnesiana, A. mearnsii, Ailanthus altissima, Lantana camara, Leucaena leucocephala and Ricinus communis.

Despite the human-driven invasions i.e. migration and transportation of goods (Mack et al. 2000), studies have shown that AIPs can 

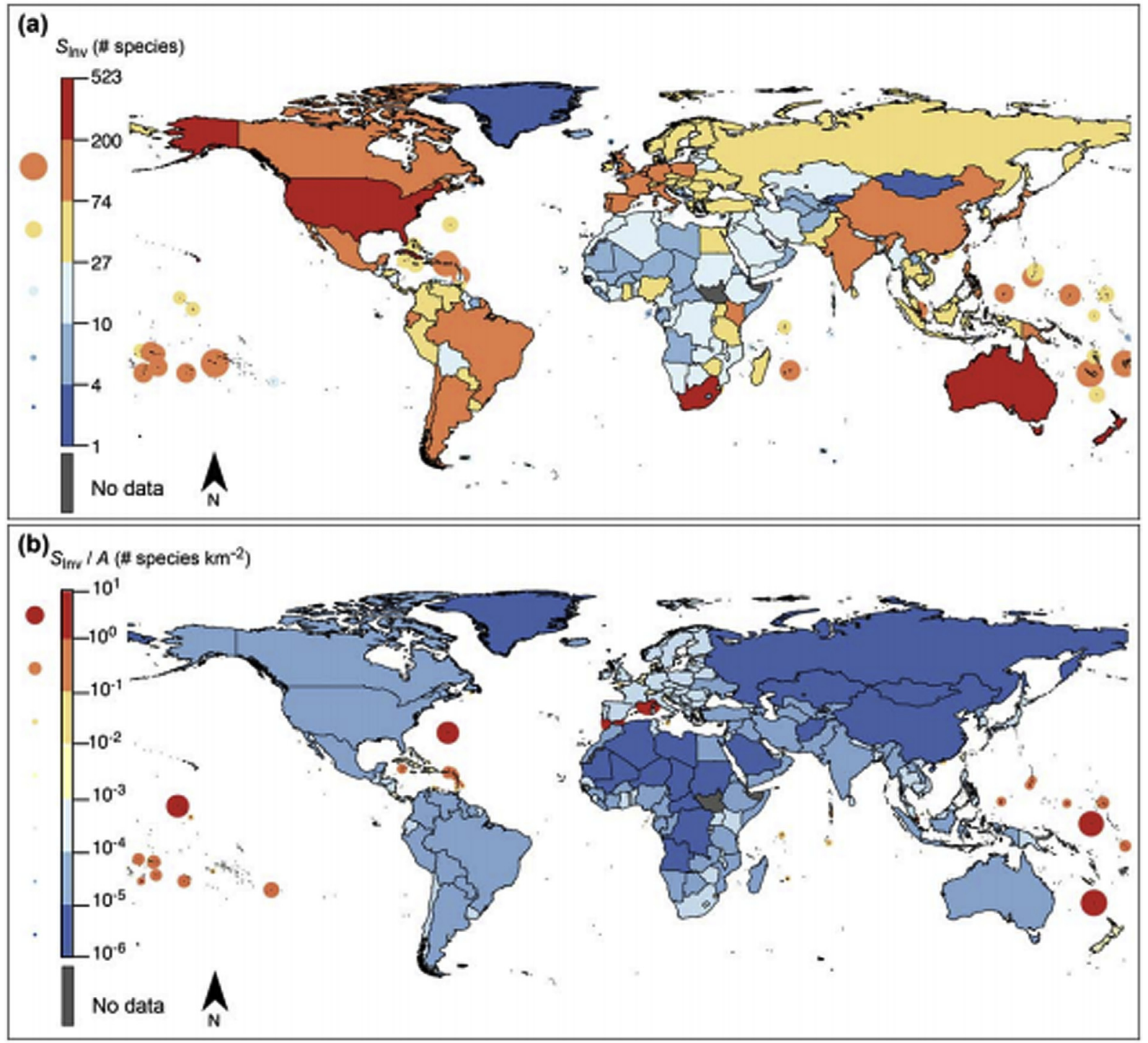

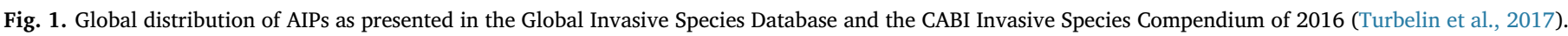
Shown are (a) the total number of recorded AIPs per country and (b) the normalized value of AIPs by the land area of each country (Turbelin et al., 2017).

spread and invade new habitats through watercourses (Dorigo et al. 2012) and birds dispersal. However, Joshi (2006) and Mack et al. (2000) noted that the human-driven invasion still remains the greatest contributing factor towards dispersion of exotic plant species globally. Dispersion of AIPs by humans can be either accidental or purposeful. Purposefully, people can introduce exotic plants in new regions for controlling other problematic species, to improve agricultural productivity, economic value and for ornamental reasons (Goodwin et al. 1999). The South American miconia (Miconia calvescens) for instance was introduced intentionally to the island of Tahiti in 1937 for ornamental reasons (Lowe et al. 2000). Other studies have recognized the impact of changing climatic conditions, as well as physiographic factors to induce invasion processes (Dark, 2004; Kriticos et al. 2003). Apart from the aforementioned forms of dispersion, the invasiveness and spreading of AIPs, generally, increases with time of habitation since their first introduction in a community (Howison, 2016).

\section{Spectral properties of AIPs in remote sensing}

The rapid spread of AIPs across the landscape renders the adoption of traditional methods of mapping AIPs such as field surveys and modelling to manage the encroachment of such species implausible, hence the need for alternative methods (Peerbhay et al. 2016c; Evangelista et al. 2009; Lass et al., 1996). Until recently, the viable alternative for detecting and mapping the spatial and temporal distribution of AIPs has relied on observing and detecting differences in their spectral reflectance using remote sensing techniques (Strand, 2007; Joshi, 2006). Several AIPs have been discriminated from their coexisting vegetation based on estimated spectral variances. These include the Bracken fern (Matongera et al. 2017; Singh et al., 2013), Bugweed (Peerbhay et al. 2015, 2016a, 2016b), Mesquite (prosopts spp.) (Robinson et al. 2016), Broom snakeweed (Gutierrezia sarothrae) (Peters et al. 1992a) and the Tickberry (Lantana camara) (Oumar 2016). Studies have revealed that plants, either alien or native, have different spectral reflectance within different regions of the electromagnetic spectrum, attributable to dissimilar biophysical (e.g. texture, canopy, leaf structure and orientation) and biochemical (e.g. chlorophyll and water content) properties of the plant ((Matongera et al. 2016a; Zhao et al. 2009). This can best be demonstrated in Fig. 1 where the thickened and succulent leaves of the Iceplant (Carpobrotus edulis) increased the absorption of its spectra around $0.9 \mu \mathrm{m}$ (see the up pointed arrow) while the dry foliage of Jubata grass (Cortaderia jubata) increased its spectral reflection around $0.55 \mu \mathrm{m}$ (see the down pointed arrow) (Strand, 2007). In these regions, the distinctiveness of spectral 


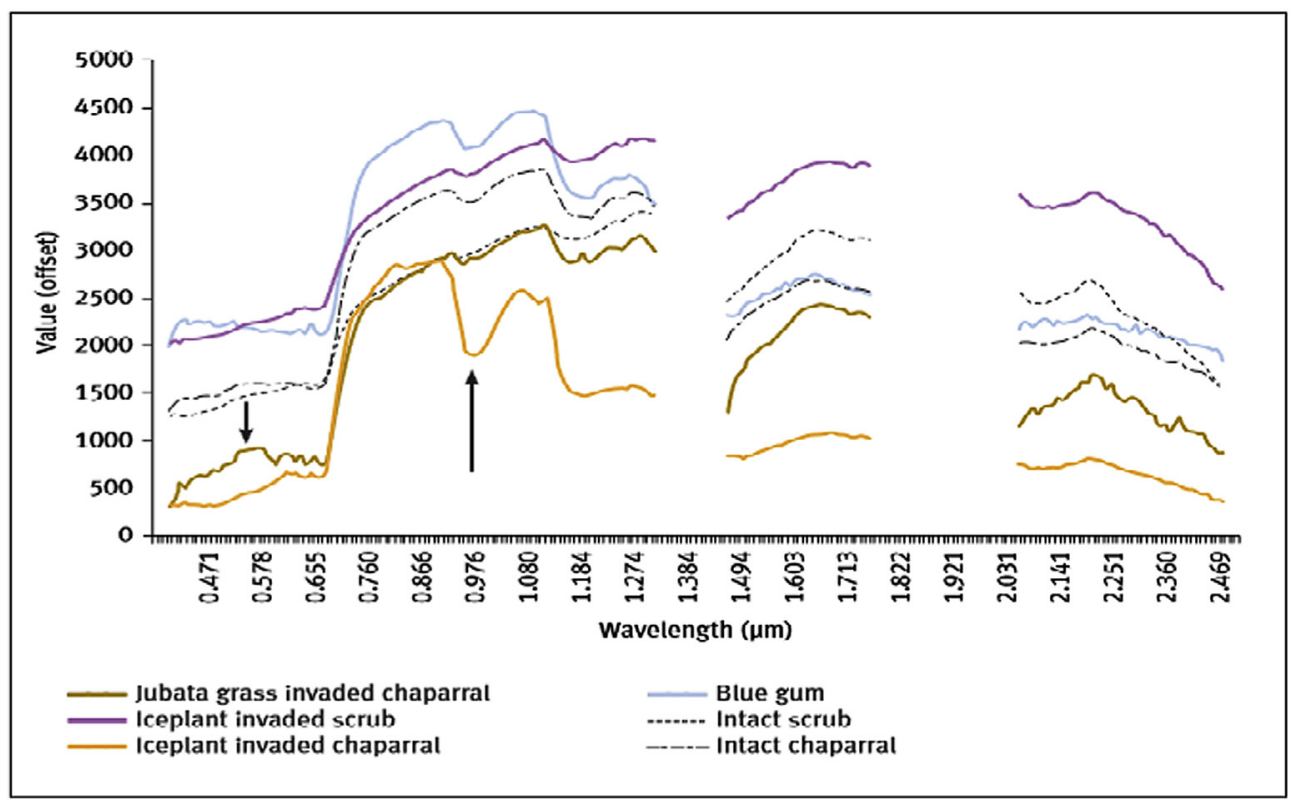

Fig. 2. Spectral signatures for different Alien Invasive Plants (AIPs). Adapted from Strand (2007).

reflectance for the two AIPs has necessitated their spectral separation from native plants using remote sensing techniques.

Like any other vegetation species, the spectral reflectance of AIPs is discreet in specific spectral wavebands in different seasons of the year especially in subtropical and temperate climatic regions (Matongera et al. 2017). For instance, a study by (Holland and Aplin, 2013) revealed that the spectral reflectance of invasive species such as bracken fern makes it difficult to discriminate bracken fern from grass during the summer season. The confusion between AIPs and grass during the summer season is due to the fact that in the visible region of the electromagnetic spectrum, most AIPs have similar reflectance with other vegetation species, which reach their peak in summer. In winter, the spectral reflectance of bracken fern is distinct from other indigenous vegetation species. A study by Page (2010) highlighted that the winter colours exhibited by bracken fern are distinct, therefore differentiating bracken fern from other vegetation types. The higher reflectance values of the majority of AIPs in winter are more evident in the red part of the spectrum due to its richness in dead material (Matongera et al. 2016a). The works of Oumar (2016) assessed the utility of SPOT 6 Sensor in detecting and mapping lantana camara in South Africa, the study revealed low reflectance in the visible spectrum and an increase in the NIR region. The low reflectance in the visible was attributed to the ability of the plant to absorb energy from the sun unlike the near-infrared region, which increases in reflectance because of low absorption.

According to Blossey and Notzold (1995), AIPs are often more vigorous and taller than co-existing vegetation due to disproportionate resource allocations. The improved physical development (i.e. vigor and height) of AIPs facilitates their discrimination from coexisting species, especially with active remote sensing sensors such as Light Detection and Ranging (LiDAR), which can effectively detect the threedimensional aspect of a feature on the ground. In an attempt to understand the invasiveness of exotic species with recognizable biological features to identify such plants, Goodwin et al. (1999) reported that differences in stem heights and flowering periods can be valuable in distinguishing AIPs from native plant species. Similarly, Everitt et al. (1995) reported that measuring plant species spectral reflectance at canopy level has been beneficial in delineating AIPs. Peerbhay et al. (2016a,b,c) further noted that AIPs often form dense infestation stands in their new habitats, facilitating their discrimination using remote sensing techniques. Generally, the phenology of AIPs is different from the indigenous plants that they normally outcompete and invade. For instance, a study by Ustin and Santos (2010) established that fennel was successfully detected and mapped when it was encroaching into grassland because the timing of its phenology was different from the grasses.

\section{Multispectral remote sensing of alien invasion}

The development of broadband remote sensing sensors such as Landsat Thematic Mapper (TM), Enhanced Thematic Mapper Plus (ETM + ), Advanced Spaceborne Thermal Emission and Reflection Radiometer (ASTER) and Satellite Pour l'Observation de la Terre (SPOT) marked the beginning of a new era in remote sensing of alien invasion (Lass et al. 2005). Generally, multispectral remote sensing sensors provide data collected between 10 and $100 \mathrm{~m}$ spatial resolution and in less than 20 bands (Huang and Asner, 2009). Many studies (e.g. Evangelista et al. 2009, Savage and Lawrence, 2010 and Viana and Aranha, 2010) have mapped and monitored AIPs using broadband coarse to medium spatial resolution data. As indicated in Table 1, the importance of these sensors includes not only the provision of large swath-width data but also repeated and free to the cost-effective dataset (Mutanga et al. 2016; Matongera et al. 2016a) which can be archived to support multi-temporal remote sensing applications. Wilfong et al. (2009), for instance, used Landsat TM images captured in November 2005 and June 2007 together with a Landsat ETM + image captured in January 2002 to detect the Amur honeysuckle (Lonicera maackii (Rupr.) Herder) in the Northeast of United States. Similarly, Evangelista et al. (2009) detected Tamarisk along the Arkansas River in Colorado using Landsat ETM + scenes acquired in April, May, June, August, September, and October.

The increased repeatability of earth observation using high temporal resolution sensors allows discerning vegetation types at different growth stages. Studies have shown that the use of images captured at different plant growing seasons is crucial for a precise detection and monitoring of changes in those plant species and in their coexisting vegetation (Hamilton et al. 2006; Joshi et al. 2004). Also, AIPs are characterized by distinctive contextual and structural features such as flowering colour and canopy structures at various phenology, which can best be detected using images taken over time. For instance, the distinctive orange-brown colour of the Chinese tamarisk (Tamarisk chinensis), before leaf shading, aided its discrimination from neighboring vegetation (Everitt et al. 1995). Furthermore, the acquisition of 


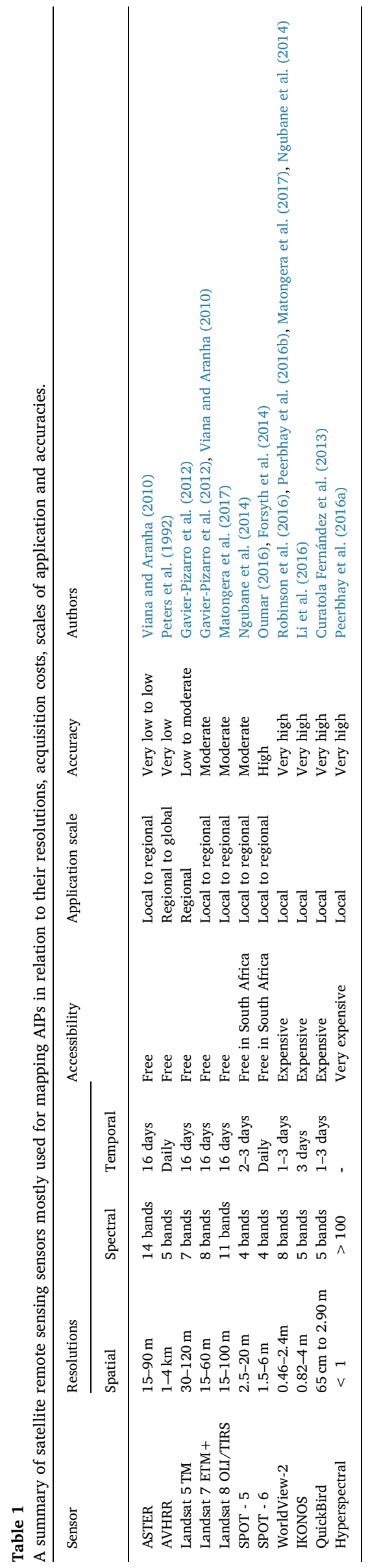

images at different plant growing seasons offer a great opportunity to compare images taken at different sun's azimuth, reducing the impact of topography and cloud shadows (Matongera et al. 2016a). In some cases, AIPs obscure background of natural vegetation particularly at early stages of their growth (Peerbhay et al. 2015). Under such conditions, images acquired over time (e.g. during non-flowering and flowering seasons) facilitate reliable mapping (Huang and Asner, 2009). On the other hand, the long-term and seasonal mapping of AIPs are vital to understand inter and intra annual distribution and abundance of such species. In addition, given that cloud cover is a challenge to satellite remote sensing, the use of multi-temporal remote sensing can optimize the acquisition of cloud-free images.

The launch of high spatial resolution multispectral sensors such as IKONOS, WorldView-2 and Quickbird are regarded a significant step towards the development of broadband sensor technology for improved detection and mapping of AIPs. For instance, Ngubane et al. (2014) reported an improved (91.67\% overall accuracy) detection of Bracken fern in Durban, South Africa, using the high spatial resolution WordView-2 than the medium spatial resolution SPOT-5 $(72.22 \%$ overall accuracy). In addition, the Pinus spp. was successfully (84\% overall accuracy) mapped by Forsyth et al. (2014) in mountainous regions of the Western Cape, using SPOT-6 imagery. Other studies that have reported an improved discrimination of vegetation types using high spatial resolution multispectral sensors include Oumar (2016), Peerbhay et al. (2016b), Li et al. (2016), Gómez-Casero et al. (2010), and Lawrence et al. (2006a). The strategically positioned bands in high spatial resolution multispectral sensors have significantly improved their performance in discrimination of vegetation types as compared to low spatial resolution multispectral sensors. Despite the improved spatial discrimination of features, literature shows that the utility of multispectral sensors in vegetation monitoring is still impeded by the poor spectral resolution (Ngubane et al., 2014).

\section{Hyperspectral remote sensing of AIPs}

To compensate for the poor spectral resolution that characterizes multispectral sensors, hyperspectral sensors emerged with hundreds of narrow contiguous spectral bands to distinguish subtle inter and intraspecies spectral variations (Atkinson et al. 2014; Cho et al. 2012). Generally, the hyperspectral dataset is collected at $2-16 \mathrm{~nm}$ spectral bandwidth across hundreds of spectral bands (Lass et al. 2002) and has been used to overcome the saturation problem commonly experienced with the adoption of multispectral sensors (Mutanga and Skidmore, 2004). This has been demonstrated by Hunt et al. (2007), who tested the potential of Airborne Visible/Infrared Imaging Spectrometer (AVIRIS) with two broadband sensors (Landsat ETM + and SPOT-4) to discriminate the Leafy spurge (Euphorbia esulaL.) near Devils Tower National Monument in Crook County, Wyoming, USA. Their findings showed a superior classification accuracy of $74 \%$ when using the hyperspectral AVIRIS in comparison to Landsat ETM + and SPOT-4, which yielded $49 \%$ and $48 \%$ overall accuracy, respectively.

Furthermore, the improved spectral resolution possible with hyperspectral dataset allows a superior classification of AIPs based on their biochemical and structural properties (Atkinson et al. 2014). With the improved spectral resolution that characterizes hyperspectral sensors, it has been possible to discern AIPs with superior accuracy, even on heterogeneous landscapes (Lawrence et al. 2006b). Other studies that have explored the utility of hyperspectral remote sensing in detection and mapping of AIPs include Peerbhay et al. (2015), He et al. (2011), Andrew and Ustin (2009), Hestir et al. (2008), Asner et al. (2008), Underwood et al. (2003) and Lass et al. (2002). Although the use of hyperspectral remote sensing has been essential in vegetation monitoring, the issue of small swath-width and high acquisition cost, as shown in Table 1, remains a challenge. Also, the huge amount of spectral information provided by hyperspectral sensors increases data dimensionality and redundancy, thereby reducing classification 
accuracy when mapping AIPs (Peerbhay et al. 2016b).

\section{The use of multisource dataset for detection of AIPs}

Data fusion or multisource dataset is increasingly being adopted for detection and mapping of AIPs using remote sensing approaches (e.g. Asner et al., 2008). Data fusion involves the integration of datasets from two or more remote sensing sensors, with various strengths and limitations (Huang and Asner, 2009). The fusion of multiple spectral, spatial and temporal properties in the same classification process push the limits of current remote sensing techniques. Peerbhay et al. (2016a), for instance, fused an AISA Eagle airborne hyperspectral dataset and a high spatial resolution WorldView-2 with LiDAR data to detect Bugweed in commercial plantation forests of KwaZulu-Natal, South Africa. Superior classification accuracies (78\% for AISA with LiDAR) and (74\% for WorldView-2 with LiDAR) were obtained with the integration of these datasets compared to $68 \%, 63 \%$ and $64 \%$ produced by AISA, WorldView-2 and LiDAR datasets alone. Similarly, Kimothi et al. (2010) optimized the detection of the Lantana camara in the Rajaji National Park of India using three Indian remote sensing sensors (IRS LISS-IV, LISS-IV and Cartosat-1) fused or in isolation. Whereas Cartosat1 produced a poor classification accuracy of $65 \%$ when used alone, its accuracy was significantly improved to $96.4 \%$ and $92.9 \%$ when fused with IRS LISS-IV and LISS-IV, respectively.

However, the full potential of data fusion for optimal detection and mapping of AIPs has not been adequately explored. Whereas, studies have shown the success of this method in detection of tree species from rangelands environment (Ghosh et al. 2014; Naidoo et al. 2012; Cho et al. 2012), the high-performance computing power required to process fused remotely sensed data makes the approach costly, especially for large-scale mapping purposes (Huang and Asner, 2009). Furthermore, data fusion for detection of AIPs has mainly been dominated by the integration of either hyperspectral or multispectral dataset with LiDAR, which is costly, hence not a viable alternative for large-scale and continuous monitoring of AIPs. On the other hand, Peerbhay et al. (2016a) suggest that the impact of Bidirectional Reflectance Distribution Function (BRDF) still needs to be addressed to minimize false classifications that can, potentially, arise because of differences in solar and sensor geometry when using multisource data.

\section{Parametric and non-parametric image classifiers for invasive alien plants}

Despite the above-mentioned factors that may influence mapping accuracy, classification algorithms remain a major factor in landscape mapping and output reliability (Lu and Weng, 2007). Broadly, image classification processes are performed using either supervised or unsupervised classification approaches (Strand, 2007; Lass et al. 2005). Image classifying algorithms could also be categorized based, among others, on either the obtainable information from the sensor, nature of the training dataset, on the basis of various parameters (Nath et al. 2014). The later could be subdivided to parametric and non-parametric image classifiers. The parametric image classifiers such as the Maximum Likelihood (MLH), Minimum Distance to Mean (MDM) and Spectral angle mapper (SAM) have been popular to enhance the discrimination of AIPs on the landscape and to reduce redundancy in remotely sensed data ( $\mathrm{Lu}$ and Weng, 2007). The advantage of these algorithms includes not only the easily accessible with almost every image classifying software but also the unsophisticated nature in the application. Although the application of these image classifiers has been successful (e.g. Ngubane et al. 2014; Curatola Fernández et al. 2013; Peters et al. 1992b), numerous challenges are reported to impair their performance. For instance, parametric image classifiers provide classification output at a pixel level (Curatola Fernández et al. 2015) and that significantly compromise the classification accuracy, especially with coarse to the medium spatial resolution multispectral dataset (Kumar and Min, 2008). Also, parametric image classifiers assume that the chosen dataset for training the classification process represents an ideal (100\%) cover of the feature or surface (Mather and Tso, 2009; Campbell and Wynne, 2011; Carson et al. 1995). Furthermore, parametric classifiers suffer from mixed pixel problem which is increased on heterogeneous landscapes (Matongera et al. 2016a; Lass et al. 2005), Hughes curse of dimensionality, Gaussian distribution of data, as well as the use of statistics to calculate class separation (Lu and Weng, 2007).

On the other hand, the non-parametric classifiers such as the Support Vector Machine (SVM), Random Forest (RF) and Artificial Neural Networks (ANN) have emerged with improved capabilities to retrieve biophysical features in vegetation. Odindi et al. (2014), tested the performance of the RF on two multispectral datasets (WV-2 and SPOT-5) with an overall classification accuracy of 84.72 and $72.22 \%$ for WV-2 and SPOT images respectively. Gavier-Pizarro et al. (2012), successfully employed the SVM to analyse the expansion of glossy privet (Ligustrum lucidum) using Landsat data in Córdoba, Argentina. Similarly, Jay et al. (2009) classified patches of the Leafy spurge in a heterogeneous rangeland of Montana in the United States, using RF classifier on a single date and time-series, with a classification accuracy varying between $72 \%$ and $95 \%$. The main advantage of non-parametric image classifiers is the ability to treat individual pixels as mixtures of pure materials and end-members in the classification process (Curatola Fernández et al. 2013). In this process, the classifiers sub-divide each individual pixel data to increase the spectral variance of different features within the pixels for superior and meaningful land cover composition as well as improved classification accuracy (Kumar and Min, 2008). As opposed to parametric classifiers that use statistics, nonparametric image classifiers such as ANN are not driven by statistical properties of the data and they are effective in extracting vegetationtype information even in heterogeneous landscapes (Gil et al. 2011). Generally, the non-parametric classifiers are suitable for classifying change than the parametric (Gavier-Pizarro et al. 2012).

\section{The use of vegetation indices for detection of AIPs}

Besides, vegetation indices, which are a ratio or linear band combinations, have been very instrumental in the mapping of AIPs (Lass et al. 2005; Gómez-Casero et al. 2010). Commonly used vegetation indices with AIPs mapping include the Normalized Difference Vegetation Index (NDVI) (Savage and Lawrence, 2010; Underwood et al. 2003), Principal Component Analysis (PCA) (Carson et al. 1995), Enhanced Vegetation Index (EVI) (Wilfong et al. 2009), Tasseled Cap (TCap) (Savage and Lawrence, 2010), Simple Ratio Nigatu et al. (Wilfong et al. 2009), Soil Adjusted Vegetation Index (Waser et al., 2008) Visible Atmospherically Resistant Index (VARI) and Normalized Difference Moisture Index (NDMI) (Wilfong et al. 2009). Wilfong et al. (2009), tested the capabilities of six vegetation indices (EVI, TCAP, SR, SAVI, VARI and NDMI) against Landsat TM and Landsat ETM+'s traditional bands in predicting the Asian Amur honeysuckle invasion in the southwest of Ohio and eastern Indiana, United States. In the study, a superior classification model (coefficient $\left(\mathrm{R}^{2}\right)$ of 0.75 for quadratic regression and 0.65 for linear regression) were achieved using NDVI. Similarly, the incorporation of the NDVI in the classification of Bracken fern on Landsat 8 and Worldview- 2 images significantly improved the mapping accuracy from $76.02 \%$ to $82.93 \%-80.08 \%$ and $87.8 \%$ for Landsat 8 and WV-2 respectively (Matongera et al. 2017). Although vegetation indices are valuable for minimizing the spectral variability caused by differences in sun viewing angles, atmospheric conditions, soil background. Mutanga and Skidmore (2004), noted that some indices such as the NDVI are affected by saturation problem, especially in high canopy densities. 


\section{Relationship between classification algorithms and remote sensing dataset}

Based on research, there is a limited literature to clearly show the synergies between the type of remotely sensed data used in conjunction with a chosen image classification algorithm. However, Nath et al. 2014, highlight that many image classification algorithms perform well on medium resolution multi-temporal data. For instance, Robinson et al. (2016), detected the invasive mesquite (Prosopis spp.) in the northwestern Pilbara, Australia using the multispectral WV-2 imagery with $80.7 \%$ overall classification accuracy. Although sensor resolutions, especially spectral and spatial, are a significant factor when discriminating vegetation types (Oumar 2016), image classification algorithms also allow the appreciation of precise separation among different plants even from averaged resolution data. For instance, Matongera et al. (2017) compared the performance of two different sensors with various spatial and spectral properties (i.e. high spatial WV-2 and medium spatial Landsat 8 OLI) in detecting Bracken fern using Discriminant Analysis. Despite the difference in resolutions of these two datasets, obtainable results reveal an insignificant or negligible difference in overall classification accuracy $(80.08 \%$ and $87.80 \%$ for Landsat 8 OLI and WV-2). The chosen image classifier significantly improved the capabilities of the medium spatial resolution Landsat 8 .

Therefore, the emergence of the new generation of multispectral sensors (e.g. SPOT-6 and Sentinel-2) is believed to further improve the detection of AIPs even by resource-limited region. This improvement in detection of AIPs will not only be due to improved sensor resolutions but rather the large swath-width and cost-effectiveness that allows repeated and operational scale monitoring of AIPs. Also, the application of robust and advanced non-parametric image classification algorithms can significantly improve the performance of these recently launched multispectral sensors. To demonstrate the role of non-parametric image classifiers over parametric classifiers, Gil et al. (2011) tested the performance of two parametric (Mahalanobis Distance and Maximum Likelihood) and non-parametric (SVM and ANN) image classifiers in assessing the potential of high-resolution satellite imagery in vegetation mapping. Although the Maximum Likelihood performed well (76.93\%) the Mahalanobis Distance performed badly with an overall accuracy of $66.04 \%$. On the other hand, both the non-parametric classifiers were successful (76.25\% and $76.95 \%$ for ANN and SVM, respectively) in spectral separation between different vegetation classes. Furthermore, the SAM performed poorly when used to classify Leafy spurge on AVIRIS, Landsat ETM + and SPOT-4 data with overall accuracies of $74 \%, 49 \%$ and $48 \%$ for AVIRIS, Landsat ETM + and SPOT-4 respectively.

\section{Challenges in remote sensing of alien invasive plants}

As aforementioned, the success of remote sensing of alien invasion relies on the identification of their unique spectral signatures facilitated by differences in biophysical and biochemical characteristics (Matongera et al. 2016a). However, when differences in these properties are not sufficiently pronounced to increase spectral variance, erroneous spectral resemblance will be recorded by the sensor for dissimilar plant species, reducing mapping accuracy of target AIPs. This is common when using broadband coarse to medium spatial resolution sensors (Huang and Asner, 2009). Also, because AIPs often grow in a mix of co-existing vegetation, their detectability can be considerably compromised, especially with averaged spectral and lower spatial resolution datasets (Matongera et al. 2016a; Huang and Asner, 2009). The large pixel sizes coupled with averaged spectral properties offered by the freely available sensor products, large swath-width and high temporal resolution sensors (see Table 1), highlights that their adoption for AIPs mapping is limited to homogenous landscapes (Hamilton et al. 2006; Carson et al. 1995; Cardina et al. 1997). Carson et al. (1995), for instance, recommends that infestation stands should be large enough or dominate the canopy to compensate for the poor spatial and spectral resolution of these sensors. Hamilton et al. (2006), indicated that a precise detection of Russian olive (Elaeagnus angustifolia L.) in central Utah was influenced by patch sizes, with small patches being underestimated or even entirely missed. According to Peerbhay et al. (2016c), the uniformity and extensiveness required when detecting the distribution of AIPs, especially with poor spatial and averaged spectral resolution datasets, is not always attainable in the newly invaded landscapes. Although these sensors provide time-series data for multitemporal AIPs mapping, valuable for improved detection (Jay et al. 2009), the success of discerning AIPs use images taken during different plant phenology depends on the availability of clear or cloud-free skies (Huang and Asner, 2009) and a precise core-registration (Singh, 1989).

Moreover, studies show that the development of satellite sensor technology is caught between balancing improvements in sensors resolutions and reducing acquisition cost while simultaneously achieving large-scale mapping of vegetation (Mutanga et al. 2016). Besides the improved mapping accuracy possible with fine resolution sensors, their value for precise mapping at an operational scale has not been fully explored (Lu and Weng, 2007). More so, the excessive acquisition costs for high spatial and hyperspectral resolution imagery is prohibitive for long-term and continuous monitoring of AIPs in countries and institutions with limited resources. The swath-width or area coverage and the application scale of fine spatial resolution multispectral and hyperspectral sensors are given in Table 1. Studies have shown that hyperspectral sensors suffer from effects of multicollinearity and multidimensionality (Gómez-Casero et al. 2010) and hence not the ultimate solution to the currently high AIPs classification accuracy problem. Therefore, it can be concluded that the current research focus of vegetation monitoring on exploration and utility of high spatial resolution and hyperspectral dataset prevent great opportunity to appreciate large-scale and long-term management of AIPs. This is likely due to the associated cost of acquiring these datasets and their small area coverage. The insignificance of this is increasingly being a problem, particularly for rangelands monitoring which is broad and extensive in extent. More so, the integration of data from different sensors does not address the small swath-width dataset provided by high spatial resolution and hyperspectral image data.

\section{Possible directions of future research}

Whereas there is considerable progress in the detection and mapping of AIPs using remotely sensed data, the full potential of this technology in estimating and mapping of AIPs has not been adequately explored. This has been demonstrated by the current trade-offs in sensor developments (i.e. resolution and acquisition costs) and application scale (Mutanga et al. 2016). Also, numerous attempts in detection and mapping of AIPs are increasingly dominated by the utility of high spatial and hyperspectral dataset (e.g. Peerbhay et al. 2016b; Peerbhay et al. 2015; Curatola Fernández et al. 2013). The acquisition costs of such dataset are prohibitive to repeated and large-scale estimation and monitoring of AIPs, particularly in countries and institutions with limited capital. Also, recent developments in remote sensing sensors technology (i.e. unmanned aerial vehicle) do not address the aforementioned issue of costs in relation to the scale of application. To optimize detection and mapping of AIPs in these regions, it is necessary to explore the capabilities of the freely available and improved spatial and spectral resolution multispectral datasets such as SPOT 6 and 7 as well as Sentinel-2 in concert with robust and advanced machine learning algorithms. The newly launched SPOT 6 and 7 provide daily coverages with improved spatial resolution (i.e. $6 \mathrm{~m}$, see Table 1), valuable for multi-temporal vegetation monitoring. On the other hand, the Sentinel-2 offers large area coverages captured at $10 \mathrm{~m}$ by $10 \mathrm{~m}$ spatial resolution, necessary for an improved operational approach in vegetation monitoring. Equally important, the advanced and robust machine learning algorithms have been valuable for vegetation 
monitoring as well as detection of AIPs. Advanced and robust machine learning algorithms such as RF and SVM can offer superior classification accuracies when mapping AIPs. However, these classifiers have not been tested with improved spatial and spectral dataset for large-scale mapping purposes. The RF and SVM have shown good mapping accuracies $(93.07 \%$ and $91.80 \%$, respectively) in mapping patterns and spatial distribution of land-use and cover types in a heterogeneous coastal landscape using RapidEye dataset (Adam et al. 2014). Therefore, it is necessary that future research endeavours focus on testing the potential of these classifiers with the recently launched multispectral dataset that is freely provided with improved spatial and spectral properties for operational scale mapping of AIPs.

\section{Conclusions}

The current study reviewed existing literature on the adoption of remotely sensed data and image classification techniques for optimal and continuous detection of AIPs at the landscape scale. The use of traditional remote sensing techniques, such as aerial photographs and averaged spectral and poor spatial resolution sensors in mapping AIPs encroachment has proved to be insufficient for improve AIPs detection and for natural resource monitoring. Equally, the use of high spatial and hyperspectral datasets does not permit long-term and wall-to-wall applications for optimal resource monitoring. Therefore, it is necessary that future studies digress slightly from the current focus on sensor spectral and spectral properties and investigate the role of image classification algorithms to improve classification output. With the launch of improved resolution data provided by the new generation multispectral sensors (i.e. Sentinel-2) and the development of non-parametric image classification algorithms (i.e. RF and SVM), optimal detection of AIPs is attainable even by resource-constrained regions. The major challenges in detection and mapping of AIPs established in this review include heterogeneity in infested landscapes, causing spectral confusion during classification and trade-offs in satellite sensor developments (i.e. costs, scale of application and accuracy) to achieve affordable wall-to-wall and continuous landscape monitoring. This technique can be tested both on single date and on time-series analysis to improve the detection of AIPs.

\section{References}

Richardson, D.M., Rejma'nek, M., 2011. Trees and shrubs as invasive alien species - a global review. Divers. Distrib. 17, 788-809.

Adam, E., Mutanga, O., Odindi, J., Abdel-Rahman, E.M., 2014. Land-use/cover classification in a heterogeneous coastal landscape using RapidEye imagery: evaluating the performance of random forest and support vector machines classifiers. Int. J. Rem. Sens. 35 (10), 3440-3458.

Aitkenhead, M., Aalders, I., 2011. Automating land cover mapping of Scotland using expert system and knowledge integration methods. Remote Sens. Environ. 115 (5), 1285-1295.

Andrew, M.E., Ustin, S.L., 2009. Habitat suitability modelling of an invasive plant with advanced remote sensing data. Divers. Distrib. 15 (4), 627-640.

Asner, G.P., Jones, M.O., Martin, R.E., Knapp, D.E., Hughes, R.F., 2008. Remote sensing of native and invasive species in Hawaiian forests. Remote Sens. Environ. 112 (5), 1912-1926.

Atkinson, J.T., Ismail, R., Robertson, M., 2014. Mapping bugweed (solanum mauritianum) infestations in pinus patula plantations using hyperspectral imagery and support vector machines. IEEE J. Sel. Top. Appl. Earth Obs. Remote Sens. 7 (1), $17-28$.

Ayele, S., 2007. The Impact of Parthenium (Parthenium Hysterophorus L.) on the Range Ecosystem Dynamics of the Jijiga Rangeland, Ethiopia. Department of Animal Sciences, School of Graduate Studies, Haramaya University, pp. 134.

Bax, N., Williamson, A., Aguero, M., Gonzalez, E., Geeves, W., 2003. Marine invasive alien species: a threat to global biodiversity. Mar. Pol. 27 (4), 313-323.

Blossey, B., Notzold, R., 1995. Evolution of increased competitive ability in invasive nonindigenous plants: a hypothesis. J. Ecol. 83 (5), 887-889.

Boyd, D.S., Foody, G.M., 2011. An overview of recent remote sensing and GIS based research in ecological informatics. Ecol. Inf. 6 (1), 25-36.

Bradley, B.A., 2014. Remote detection of invasive plants: a review of spectral, textural and phenological approaches. Biol. Invasions 16 (7), 1411-1425.

Campbell, J.B., Wynne, R.H., 2011. Introduction to Remote Sensing. Guilford Press.

Cardina, J., Johnson, G.A., Sparrow, D.H., 1997. The nature and consequence of weed spatial distribution. Weed Sci. 364-373.
Carson, H.W., Lass, L.W., Callihan, R.H., 1995. Detection of Yellow Hawkweed (Hieracium Pratense) with High Resolution Multispectral Digital Imagery. Weed Technology, pp. 477-483.

Cho, M.A., Mathieu, R., Asner, G.P., Naidoo, L., van Aardt, J., Ramoelo, A., Debba, P., Wessels, K., Main, R., Smit, I.P., 2012. Mapping tree species composition in South African savannas using an integrated airborne spectral and LiDAR system. Remote Sens. Environ. 125, 214-226.

Crossman, N.D., Kochergen, J., 2002. Mapping Environmental Weeds in the Mount Lofty Ranges, South Australia, Using High Resolution Infrared Aerial Photography. Plant Protection Society of WA Incorporated.

Curatola Fernández, G., Silva, B., Gawlik, J., Thies, B., Bendix, J., 2013. Bracken fern frond status classification in the Andes of southern Ecuador: combining multispectral satellite data and field spectroscopy. Int. J. Rem. Sens. 34 (20), 7020-7037.

Curatola Fernández, G.F., Obermeier, W.A., Gerique, A., López Sandoval, M.F., Lehnert, L.W., Thies, B., Bendix, J., 2015. Land cover change in the andes of southern Ecuador-patterns and drivers. Rem. Sens. 7 (3), 2509-2542.

Dark, S.J., 2004. The biogeography of invasive alien plants in California: an application of GIS and spatial regression analysis. Divers. Distrib. 10 (1), 1-9.

Dorigo, W., Lucieer, A., Podobnikar, T., Čarni, A., 2012. Mapping invasive Fallopia japonica by combined spectral, spatial, and temporal analysis of digital orthophotos. Int. J. Appl. Earth Obs. Geoinf. 19, 185-195.

Evangelista, P.H., Stohlgren, T.J., Morisette, J.T., Kumar, S., 2009. Mapping invasive tamarisk (Tamarix): a comparison of single-scene and time-series analyses of remotely sensed data. Rem. Sens. 1 (3), 519-533.

Everitt, J.H., Escobar, D.E., Davis, M.R., 1995. Year: Published. Using Remote Sensing for Detecting and Mapping Noxious Plants. Weed Abstracts, pp. 639-649.

Everitt, J.H., Escobar, D.E., Alaniz, M.A., Davis, M.R., Richerson, J.V., 1996. Using Spatial Information Technologies to Map Chinese Tamarisk (Tamarix Chinensis) Infestations. Weed Sci. pp. 194-201.

Flood, N., 2013. Seasonal composite Landsat TM/ETM + images using the Medoid (a multi-dimensional median). Rem. Sens. 5 (12), 6481-6500.

Forsyth, A.T., Gibson, L.A., Turner, A.A., 2014. Assessment of SPOT 6 imagery for mapping the invasive alien plant species Pinus spp. In: A Mountainous Area of the Western Cape.

Foxcroft, L.C., Richardson, D.M., Wilson, J.R., 2008. Ornamental plants as invasive aliens: problems and solutions in Kruger National Park, South Africa. Environ. Manag. 41 (1), 32-51.

Gaertner, M., Den Breeyen, A., Hui, C., Richardson, D.M., 2009. Impacts of alien plant invasions on species richness in Mediterranean-type ecosystems: a meta-analysis. Prog. Phys. Geogr. 33 (3), 319-338.

Gavier-Pizarro, G.I., Kuemmerle, T., Hoyos, L.E., Stewart, S.I., Huebner, C.D., Keuler, N.S., Radeloff, V.C., 2012. Monitoring the invasion of an exotic tree (Ligustrum lucidum) from 1983 to 2006 with Landsat TM/ETM + satellite data and Support Vector Machines in Córdoba, Argentina. Remote Sens. Environ. 122, 134-145.

Ghosh, A., Fassnacht, F.E., Joshi, P., Koch, B., 2014. A framework for mapping tree species combining hyperspectral and LiDAR data: role of selected classifiers and sensor across three spatial scales. Int. J. Appl. Earth Obs. Geoinf. 26, 49-63.

Gil, A., Yu, Q., Lobo, A., Lourenço, P., Silva, L., Calado, H., 2011. Assessing the effectiveness of high resolution satellite imagery for vegetation mapping in small islands protected areas. J. Coast Res. 64, 1663-1667.

Gómez-Casero, M.T., Castillejo-González, I., García-Ferrer, A., Peña-Barragán, J.M., Jurado-Expósito, M., García-Torres, L., López-Granados, F., 2010. Spectral discrimination of wild oat and canary grass in wheat fields for less herbicide application. Agron. Sustain. Dev. 30 (3), 689-699.

Goodwin, B.J., McAllister, A.J., Fahrig, L., 1999. Predicting invasiveness of plant species based on biological information. Conserv. Biol. 13 (2), 422-426.

Gurevitch, J., Padilla, D.K., 2004. Are invasive species a major cause of extinctions? Trends Ecol. Evol. 19 (9), 470-474.

Hamilton, R., Megown, K., Lachowski, H., Campbell, R., 2006. Mapping Russian Olive: Using Remote Sensing to Map an Invasive Tree. US Department of Agriculture Forest Service Remote Sensing Application Center RSAC-0087-RPT1. http://www.fs.fed.us/ eng/rsac/invasivespecies/documents/0087-RPT1.pdf, Accessed date: 15 September 2011.

He, K.S., Rocchini, D., Neteler, M., Nagendra, H., 2011. Benefits of hyperspectral remote sensing for tracking plant invasions. Divers. Distrib. 17 (3), 381-392.

Hestir, E.L., Khanna, S., Andrew, M.E., Santos, M.J., Viers, J.H., Greenberg, J.A., Rajapakse, S.S., Ustin, S.L., 2008. Identification of invasive vegetation using hyperspectral remote sensing in the California Delta ecosystem. Remote Sens. Environ. 112 (11), 4034-4047.

Higgins, S.I., Richardson, D.M., Cowling, R.M., Trinder-Smith, T.H., 1999. Predicting the landscape-scale distribution of alien plants and their threat to plant diversity. Conserv. Biol. 13 (2), 303-313.

Holland, J., Aplin, P., 2013. Super-resolution image analysis as a means of monitoring bracken (Pteridium aquilinum) distributions. ISPRS J. Photogrammetry Remote Sens. 75, 48-63.

Howison, O.E., 2016. How Do Alien Plants Invade: an Analysis of the Historical Spread and Potential Distribution of the Invasive Alien Plant Chromolaena Odorata in and Around Hluhluwe Game Reserve.

Huang, C.-y., Asner, G.P., 2009. Applications of remote sensing to alien invasive plant studies. Sensors 9 (6), 4869-4889.

Hunt, E.R., Daughtry, C.S., Kim, M.S., Williams, A.E.P., 2007. Using canopy reflectance models and spectral angles to assess potential of remote sensing to detect invasive weeds. J. Appl. Remote Sens. 1 (1), 013506 013506-19.

Jay, S., Lawrence, R., Repasky, K., Keith, C., 2009. Invasive species mapping using low cost hyper spectral imagery. Year: Published. ASPRS (1), 365-373 Annual Conference, 
Joshi, C., 2006. Mapping Cryptic Invaders and Invisability of Tropical Forest Ecosystems: Chromolaena Odorata in Nepal. PhD Thesis. ITC, Enschede, Netherlands.

Joshi, C., de Leeuw, J., van Duren, I.C., 2004. Remote sensing and GIS applications for mapping and spatial modelling of invasive species. Year: Published. Proc. ISPRS. B7.

Kalusová, V., Chytrý, M., Kartesz, J.T., Nishino, M., Pyšek, P., 2013. Where do they come from and where do they go? European natural habitats as donors of invasive alien plants globally. Divers. Distrib. 19 (2), 199-214.

Karki, D., 2009. Ecological and Socio-economic Impacts of Parthenium Hysterophorus L. Invasion in Two Urban Areas in Nepal. Department of Botany, Tribhuvan University.

Kimothi, M., Dasari, A., 2010. Methodology to map the spread of an invasive plant (Lantana camara L.) in forest ecosystems using Indian remote sensing satellite data. Int. J. Rem. Sens. 31 (12), 3273-3289.

Kimothi, M., Anitha, D., Vasistha, H., Soni, P., Chandola, S., 2010. Remote sensing to map the invasive weed, Lantana camara in forests. Trop. Ecol. 51 (1), 67-74.

Kriticos, D., Sutherst, R., Brown, J., Adkins, S., Maywald, G., 2003. Climate change and the potential distribution of an invasive alien plant: Acacia nilotica ssp. indica in Australia. J. Appl. Ecol. 40 (1), 111-124.

Kumar, A., Min, H., 2008. Some issues related with sub-pixel classification using Hyperion data. Proc. Int. Archiv. Photogram. Remote Sens. Spat. Inf. Sci. 37, 249-254.

Lass, L.W., Carson, H.W., Callihan, R.H., 1996. Detection of Yellow Starthistle (Centaurea Solstitialis) and Common St. Johnswort (Hypericum perforatum) with Multispectral Digital Imagery. Weed Technology, pp. 466-474.

Lass, L.W., Thill, D.C., Shafii, B., Prather, T.S., 2002. Detecting spotted knapweed (centaurea maculosa) with hyperspectral remote sensing technology 1. Weed Technol. 16 (2), 426-432.

Lass, L.W., Prather, T.S., Glenn, N.F., Weber, K.T., Mundt, J.T., Pettingill, J., 2005. A review of remote sensing of invasive weeds and example of the early detection of spotted knapweed (Centaurea maculosa) and babysbreath (Gypsophila paniculata) with a hyperspectral sensor. Weed Sci. 53 (2), 242-251.

Lawrence, R., Hurst, R., Weaver, T., Aspinall, R., 2006a. Mapping prairie pothole communities with multitemporal IKONOS satellite imagery. Photogramm. Eng. Rem. Sens. 72 (2), 169-174.

Lawrence, R.L., Wood, S.D., Sheley, R.L., 2006b. Mapping invasive plants using hyperspectral imagery and Breiman Cutler classifications (RandomForest). Remote Sens. Environ. 100 (3), 356-362.

Le Maitre, D.C., van Wilgen, B.W., Gelderblom, C., Bailey, C., Chapman, R.A., Nel, J., 2002. Invasive alien trees and water resources in South Africa: case studies of the costs and benefits of management. For. Ecol. Manag. 160 (1), 143-159.

Li, J., Liu, Y., Mo, C., Wang, L., Pang, G., Cao, M., 2016. IKONOS image-based extraction of the distribution area of Stellera chamaejasme L. In Qilian county of Qinghai Province, China. Rem. Sens. 8 (2), 148.

Lowe, S., Browne, M., Boudjelas, S., De Poorter, M., 2000. 100 of the World's Worst Invasive Alien Species: a Selection from the Global Invasive Species Database.

Lu, D., Weng, Q., 2007. A survey of image classification methods and techniques for improving classification performance. Int. J. Rem. Sens. 28 (5), 823-870.

Mack, R.N., Simberloff, D., Mark Lonsdale, W., Evans, H., Clout, M., Bazzaz, F.A., 2000. Biotic invasions: causes, epidemiology, global consequences, and control. Ecol. Appl. 10 (3), 689-710.

Mather, P., Tso, B., 2009. Classification Methods for Remotely Sensed Data. CRC press, Boca Raton.

Matongera, T.N., Mutanga, O., Dube, T., Lottering, R.T., 2016a. Detection and mapping of bracken fern weeds using multispectral remotely sensed data: a review of progress and challenges. Geocarto Int. 1-16.

Matongera, T.N., Mutanga, O., Dube, T., Lottering, R.T., 2016b. Detection and mapping of bracken fern weeds using multispectral remotely sensed data: a review of progress and challenges. Geocarto Int. 1-31 (just-accepted).

Matongera, T.N., Mutanga, O., Dube, T., Sibanda, M., 2017. Detection and mapping the spatial distribution of bracken fern weeds using the Landsat 8 OLI new generation sensor. Int. J. Appl. Earth Obs. Geoinf. 57, 93-103.

McConnachie, A., Strathie, L., Mersie, W., Gebrehiwot, L., Zewdie, K., Abdurehim, A., Abrha, B., Araya, T., Asaregew, F., Assefa, F., 2011. Current and potential geographical distribution of the invasive plant Parthenium hysterophorus (Asteraceae) in eastern and southern Africa. Weed Res. 51 (1), 71-84.

Mutanga, O., Skidmore, A.K., 2004. Narrow band vegetation indices overcome the saturation problem in biomass estimation. Int. J. Rem. Sens. 25 (19), 3999-4014.

Mutanga, O., Dube, T., Ahmed, F., 2016. Progress in remote sensing: vegetation monitoring in South Africa. S. Afr. Geogr. J. 98 (3), 461-471.

Naidoo, L., Cho, M., Mathieu, R., Asner, G., 2012. Classification of savanna tree species, in the Greater Kruger National Park region, by integrating hyperspectral and LiDAR data in a Random Forest data mining environment. ISPRS J. Photogrammetry Remote Sens. 69, 167-179.

Nath, S.S., Mishra, G., Kar, J., Chakraborty, S., Dey, N., 2014. A survey of image classification methods and techniques. Year: Published In: Control, Instrumentation, Communication and Computational Technologies (ICCICCT), 2014 International Conference on. IEEE, pp. 554-557.

Ngubane, Z., Odindi, J., Mutanga, O., Slotow, R., 2014. Assessment of the contribution of WorldView-2 strategically positioned bands in Bracken fern (Pteridium aquilinum (L.) Kuhn) mapping. S. Afr. J. Geol. 3 (2), 210-223.

Nigatu, L., Hassen, A., Sharma, J., Adkins, S.W., 2010. Impact of Parthenium hysterophorus on grazing land communities in north-eastern Ethiopia. Weed Biol. Manag. 10 (3), 143-152.
Niphadkar, M., Nagendra, H., 2016. Remote sensing of invasive plants: incorporating functional traits into the picture. Int. J. Rem. Sens. 37 (13), 3074-3085.

Odindi, J., Adam, E., Ngubane, Z., Mutanga, O., Slotow, R., 2014. Comparison between WorldView-2 and SPOT-5 images in mapping the bracken fern using the random forest algorithm. J. Appl. Remote Sens. 8 (1) 083527-083527.

Oumar, Z., 2016. Assessing the utility of the spot 6 sensor in detecting and mapping Lantana camara for a community clearing project in KwaZulu-Natal, South Africa. S. Afr. J. Geol. 5 (2), 214-226.

Page, C.N., 2010. The Ferns of Britain and Ireland, third ed. Cambridge University, Press.

Peerbhay, K.Y., Mutanga, O., Ismail, R., 2015. Random forests unsupervised classification: the detection and mapping of solanum mauritianum infestations in plantation forestry using hyperspectral data. IEEE J. Sel. Top. Appl. Earth Obs. Remote Sens. 8 (6), 3107-3122.

Peerbhay, K., Mutanga, O., Lottering, R., Bangamwabo, V., Ismail, R., 2016a. Detecting bugweed (Solanum mauritianum) abundance in plantation forestry using multisource remote sensing. ISPRS J. Photogrammetry Remote Sens. 121, 167-176.

Peerbhay, K., Mutanga, O., Lottering, R., Ismail, R., 2016b. Mapping Solanum mauritianum plant invasions using WorldView-2 imagery and unsupervised random forests. Remote Sens. Environ. 182, 39-48.

Peerbhay, K.Y., Mutanga, O., Ismail, R., 2016c. The identification and remote detection of alien invasive plants in commercial forests: an Overview. S. Afr. J. Geol. 5 (1), 49-67.

Pejchar, L., Mooney, H.A., 2009. Invasive species, ecosystem services and human wellbeing. Trends Ecol. Evol. 24 (9), 497-504.

Peters, A.J., Reed, B.C., Eve, M.D., McDaniel, K.C., 1992a. Remote Sensing of Broom Snakeweed (Gutierrezia Sarothrae) with NOAA-10 Spectral Image Processing. Weed Technol, pp. 1015-1020.

Peters, A.J., Reed, B.C., Eve, M.D., McDaniel, K.C., 1992b. Remote sensing of broom snakeweed (Gutierrezia sarothrae) with NOAA-10 spectral image processing. Weed Technol. 6, 10151015.

Pimentel, D., Zuniga, R., Morrison, D., 2005. Update on the environmental and economic costs associated with alien-invasive species in the United States. Ecol. Econ. 52 (3), 273-288.

Pyšek, P., 1998. Is there a taxonomic pattern to plant invasions? Oikos 282-294.

Robinson, T., Wardell-Johnson, G., Pracilio, G., Brown, C., Corner, R., Van Klinken, R., 2016. Testing the discrimination and detection limits of WorldView-2 imagery on a challenging invasive plant target. Int. J. Appl. Earth Obs. Geoinf. 44, 23-30.

Rocchini, D., Andreo, V., Förster, M., Garzon-Lopez, C.X., Gutierrez, A.P., Gillespie, T.W., Hauffe, H.C., He, K.S., Kleinschmit, B., Mairota, P., 2015. Potential of remote sensing to predict species invasions: a modelling perspective. Prog. Phys. Geogr. 39 (3), 283-309.

Savage, S.L., Lawrence, R.L., 2010. Vegetation dynamics in yellowstone's northern range: 1985 to 1999. Photogramm. Eng. Rem. Sens. 76 (5), 547-556.

Shackleton, C., Shackleton, S., Buiten, E., Bird, N., 2007. The importance of dry woodlands and forests in rural livelihoods and poverty alleviation in South Africa. Forest Policy Econ 9 (5), 558-577.

Shezi, M.I.Z., Poona, M.N.K., 2010. UKZN/DAEA.

Singh, A., 1989. Review article digital change detection techniques using remotely-sensed data. Int. J. Rem. Sens. 10 (6), 989-1003.

Singh, K., Forbes, A., Akombelwa, M., 2013. The evaluation of high resolution aerial imagery for monitoring of bracken fern. S. Afr. J. Geol. 2 (4), 296208.

Strand, H., 2007. Sourcebook on Remote Sensing and Biodiversity Indicat Montreal; Convention on Biological Diversityors.

Swayne, D. A., Yang, W., Voinov, A., Rizzoli, A. \& Filatova, T. Remote Sensing Time Series for Modeling Invasive Species Distribution: a Case Study of Tamarix Spp. In the US and Mexico.

Todaro, M.P., 1969. A model of labor migration and urban unemployment in less developed countries. Am. Econ. Rev. 59 (1), 138-148.

Turbelin, A.J., Malamud, B.D., Francis, R.A., 2017. Mapping the global state of invasive alien species: patterns of invasion and policy responses. Global Ecol. Biogeogr. 26, 78-92.

Underwood, E., Ustin, S., DiPietro, D., 2003. Mapping nonnative plants using hyperspectral imagery. Remote Sens. Environ. 86 (2), 150-161.

Ustin, S.L., Santos, M., 2010. Spectral identification of native and non-native plant species. In: Proceedings of ASD and IEEEGRS; Art, Science and Applications of Reflectance Spectroscopy Symposium.

Viana, H., Aranha, J., 2010. Mapping invasive species (Acacia dealbata link) using ASTER/TERRA and LANDSAT 7 ETM + imagery. Forest Landscapes and global changenew Frontiers in management, Conservation and restoration. Year: Published In: Proceedings of the IUFRO Landscape Ecology Working Group International Conference.

Waser, L., Baltsavias, E., Ecker, K., Eisenbeiss, H., Feldmeyer-Christe, E., Ginzler, C., Küchler, M., Zhang, L., 2008. Assessing changes of forest area and shrub encroachment in a mire ecosystem using digital surface models and CIR aerial images. Remote Sens. Environ. 112 (5), 1956-1968.

Wilfong, B.N., Gorchov, D.L., Henry, M.C., 2009. Detecting an invasive shrub in deciduous forest understories using remote sensing. Weed Sci. 57 (5), 512-520.

Zhao, B., Yan, Y., Guo, H., He, M., Gu, Y., Li, B., 2009. Monitoring rapid vegetation succession in estuarine wetland using time series MODIS-based indicators: an application in the Yangtze River Delta area. Ecol. Indicat. 9 (2), 346-356.

Zuberi, M.I., Gosaye, T., Hossain, S., 2014. Potential threat of alien invasive species: parthenium hysterophorus L. to subsistence agriculture in Ethiopia. Sarhad J. Agric. 30 (1), $117-125$ 